\title{
A Biomechanical Paradigm Shift: Part I: Transforming Lower Extremity Biomechanics Terminology, Nomenclature and Science as an Upgrade to "STJ Neutral" and “Normal" Biomechanics
}

\author{
Dennis Shavelson* \\ The Foot Typing Center@LifeStyle Podiatry, USA \\ *Corresponding author: Dennis Shavelson, DPM, The Foot Typing Center@LifeStyle Podiatry, NYC, USA
}

Submission: June 05, 2018; Published: June 13, 2018

\section{Hypothesis}

Biomechanical Obstructions to Biomechanical Research and Practice need to be enshrined as Iconic Precursors and Replaced with a Paradigm Shift in order to allow Lower Extremity Biomechanics to Acculturate Onwards and Upwards as an Evidence Based Science.

\section{Mission Bullets}

A. Let's lay to rest current belief that custom foot orthotics (CFO's) work independently or medicinally. At their useful best, custom foot orthotics are a prop that can foster the body to adapt to new architectural and force stresses that force more optimal stability, support, strength, symmetry and balance when underfoot the right subject.

B. Let's admit that current mainstream custom foot orthotics as a stand-alone product do not "Fix" or "Correct" anything. Picture a person putting CFO's underfoot and then never moving, exercising or training.

C. Let's enshrine the iconic biomechanical terminology and nomenclature (that has served its purpose) that is now inert, unscientific, poorly defined, poorly evidenced, poorly understood that is acting as a wedge to growth of the science. Let's start with jettisoning terminology like subtalar joint neutral position \& casting, forefoot valgus, forefoot varus, pronation, hyperpronation, the arches of the foot, normal and neutral in no special order when it comes to biomechanics and orthotics. For decades, there have been few if any high level peer reviewed clinically applicable additions to the literature. This is due to the inability for researchers to develop cohorts that produce evidence that is clinically viable and applicable and the lack of consensus surrounding CFO's in the biomedical engineering community.

D. Let's enshrine the iconic "wet test" when it comes to foot typing and develop a foot typing method that classifies all feet into subgroups that can be researched foot type-specific in order to develop better cohorts.

E. Let's enshrine as iconic the current goals/ceiling for researching and marketing custom foot orthotics such as "complaint relief", they make one "feel good" and "they work".

F. Let's set the biomechanical goals/ceiling for custom foot orthotics higher by installing and marketing nomenclature like "improving functionality", making muscle engines better leveraged.

G. Let's eliminate the less than iconic unproven and exaggerated myths and false promises regarding custom foot orthotics. Terminology regarding CFO's such as "they place feet in healthy or optimal positions" they "slow down bunion progression", they "correct pronation", they "enhance athletic performance", they "improve functioning of muscles" they "reduce pathological movement", they "enhance foot support" and especially the anti-Newtonian myth that current custom foot orthotics "realign and position feet properly" should be ensconced forever.

H. Let's upgrade our goal set when it comes to CFO's to maintaining and improving weight bearing stance, optimizing body support and improving body movement and performance, injury free, efficiently". The myths listed above that are being disproven in the literature depend on the definition of a custom foot orthotic to be a device that reduces abnormal motion or abnormal position of the foot. A device that controls abnormal foot functions and/or accommodates painful areas of the foot as a standalone product. These claims are myths because an orthotic can never be a stand-alone product unless it is acting as an accommodative device such as a crutch, cane or walker with a heroic goal to maintain already poor biomechanical architecture and engineering at an accepted low level [1]. 


\section{The Givens}

a. The underutilized tenets with biomechanical architecture and engineering of the lower extremity is that there are three constant forces (the "crippling constants" in a civilized society), Earth's gravity, ground reaction force (grf) and the atrophic and deforming pressures of civilized shoes (Figure 1) whose stresses must be adapted to from birth until death successfully as a given when developing a dynamic force equilibrium for living our weight bearing lives upright and bipedal.

b. Custom Foot Orthotics cannot supply, as stand-alone devices, enough force to accomplish this.

c. In closed chain upright stance and function the most important locations of the body needing optimal stability, support, strength, symmetry and balance are the feet.

d. Once the degenerative/draining (as suggested, changed from negative as more descriptive) energy of the crippling constants is neutralized with muscle engine power, the body must then have available additional energy in order to move about, lift objects and perform tasks and absorb additional stress supplied by internal and external factors such as additional body weight and inclines and obstacles within to ground surface.

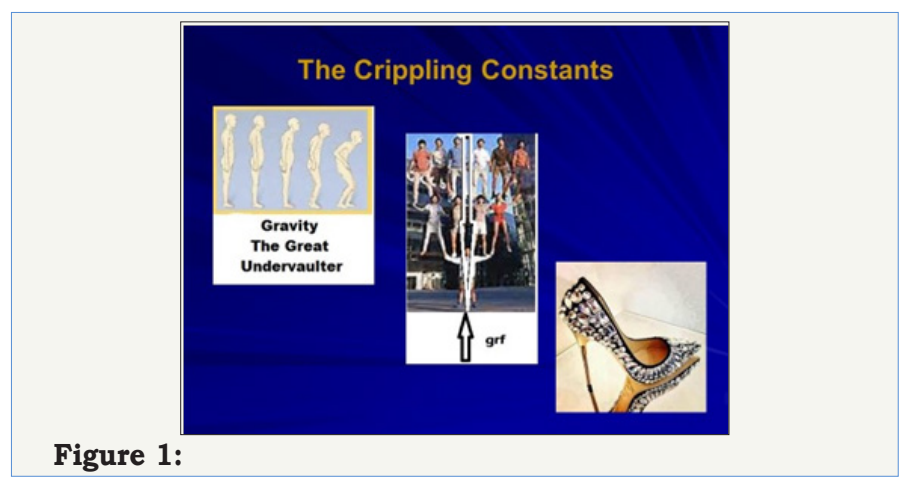

\section{The Current Biomechanical Force Equilibrium Formula}

Force of the Crippling Constants+Force of Standing, Moving and Performing Tasks+Force of Internal and External Factors=The Forces Produced/Available to Perform Injury, Deformity, Degeneration Free, Efficiently

When the force produced/available internally is insufficient to offset the stresses produced by the crippling constants and those emanating from living life in closed chain, injury, deformity, degeneration and poor performance are the result.

\section{Fact}

Current nonsurgical biomechanics and custom foot orthotics cannot produce [2-8] the force needed to overcome these constants nor can they stimulate adaptation of the muscle engines of the body to help provide the additional force to increase the force produced/ available internally in the equation.

\section{Hypothesis}

The Conversion of a Custom Foot Orthotic from a Fix-It-Product (Stand Alone) to a Make-the-Body-Fixable-Product (A Prop) May be the Answer. We need to have a foundational pedal posture and a neuromuscular system that is both optimally structured and mechanically engineered that it will produce enough internal force to create a dynamic force equilibrium formula for living life upright and active healthier, longer, holistically.

Let's begin with a new Biomechanical Force Equilibrium Formula:

Force of the Crippling Constants+Force of Standing, Moving and Performing Tasks+Force of Internal and External Factors=The Force Produced/Available Internally+the Force of Props+the Force of Therapy, Training and Coaching.

Is there literature describing the function of props? Not that I am aware. There are noncommercial publications with pictures or illustrations of prop usage, but there is not a descriptive reference. However, let's take an overview of prop function. The way CFO props are used architecturally will depend upon the clinical experience, the ability to develop teams of biomechanically oriented practitioners and researchers willing to acculturate to a new paradigm. Utilizing props (straps, pads, OTC orthotics and CFO's) is the safest way to teach your body to achieve proper alignment in any pose. Props free one up to master tougher poses, to control stability and flexibility internally safely and efficiently (Figure 2 ). Then to be weaned away, props are the way to offset the crippling constants thereby leaving existing internal architecture and engineering to be prodded towards more optimal structural and functional force equilibrium (think orthodontics optimizing the structure and function of the arches of the mouth).

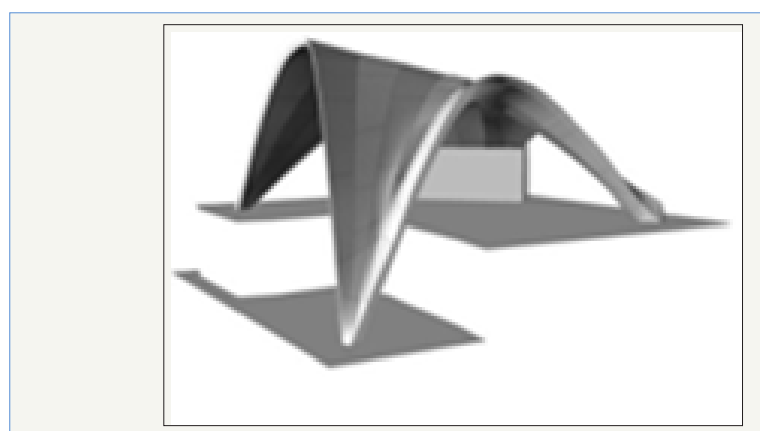

Figure 2:

\section{Summary}

The focus of biomechanics should change from being complaint oriented to being oriented to provide optimal positioning and function.

\section{Discussion}

Biomechanical props, Movement Training and Therapy and Foot Surgery play important yet distinct roles during one's biomechanical lifetime, not one or the other living in separate vacuums without collaboration. This can happen with and without walls eventually becoming skilled maintenance as cohorts live their lives. The foot poses differently thousands of times a day at different times in stance and gait with its base of support optimally placed under the heel, the first metatarsal and the fifth metatarsal. Dynamic posing sometimes places feet and their tissues in stressful 
poses (either collapsed or too stable) that if allowed to exist lead to predictable injury, deformation, degeneration, weakness, stiffness and poor performance. The foot must move from and be returned to what in athletic disciplines is called "a ready, optimal or critical position" from which it is best suited to perform its static and functional tasks [9-13].

\section{Whole Body Compensation}

Due to proprioceptive and muscle twitch nerves, the central nervous system (CNS), the myofascia and the body's ability to use its muscle engines perform and compensate in various locations along the posture for supportive and physical needs while maintaining structural integrity. We have built in sensors, a computer and engines wired together. The problem is that once any area within the posture fails to be well posed or perform optimally, other areas adjust for the good of the whole body. A problem in one location of the body produces problems in other body locations as compensation. The areas of the body that fail most often are the weight bearing joints of the lower extremity, led by the feet and lower back. The Dystopian Posture starts in the feet and goes up the postural chain over time, with increased weight and increased activity (Figure 3).

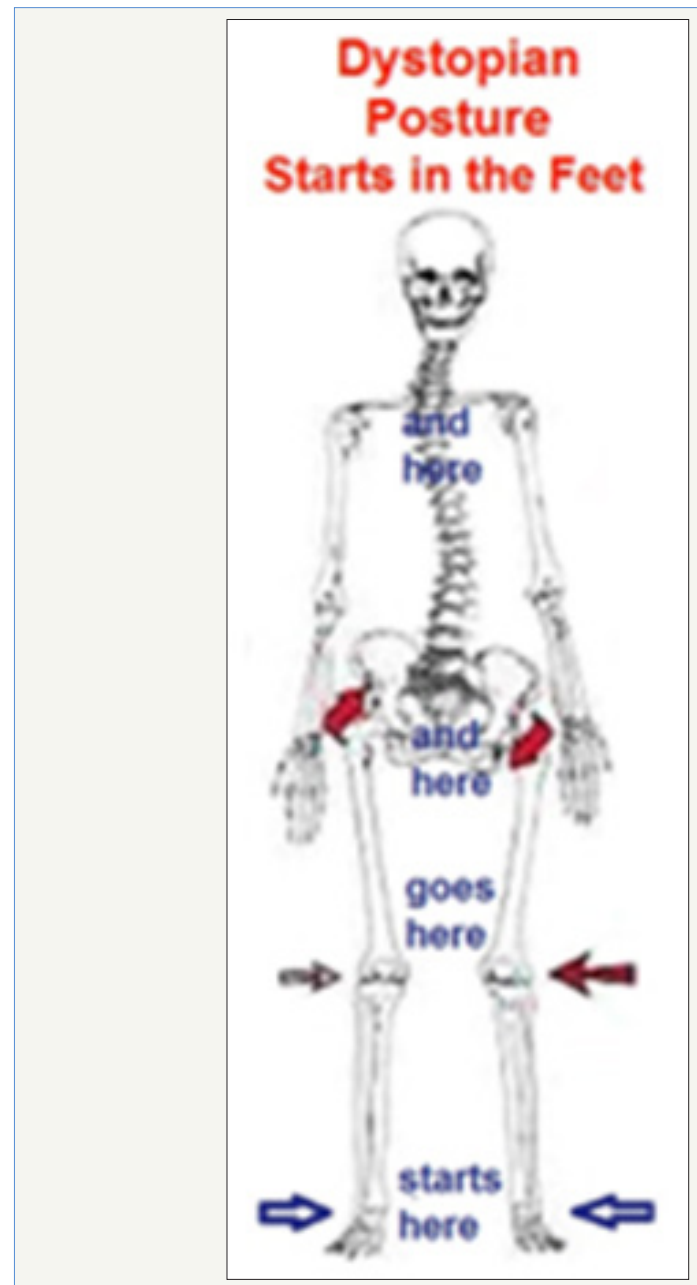

Figure 3:

Because of this fact, there is no "normal" position for any joint, theoretical or otherwise in biomechanics. There is no single neutral position for any joint, theoretical or otherwise in biomechanics. This is because the optimal position of any weight bearing joint is determined, among other factors by the biomechanics of the body as a whole. The best we can define "normal' is to say that biological structures that are impacted by Wolff's and Davis's Laws in closed chain (i.e. the joints, the bones or the muscle engines) theoretically function within a range of motion that resists tissue stress and deformation in all directions. These tissues would be optimized throughout the biomechanical timeline of that body $[14,15]$.

The theoretical goal of this hypothetical paper is to expand and organize the diagnosis and treatment of the architecture, morphology and engineering of the foot by considering eliminating old language and methods with new ones and then trying to develop consensus among those working biomechanically. As an example, I am considering replacing terminology normal and neutral with optimal or critical. In reality, as we optimize a joint in a closed chain system, we create tissue stress in other joints within that system. As we function during each day, the axis about which our joints and muscle engines perform and their health states changes constantly obeying biological as well as mechanical laws.

\section{The Foot as the Foundation of Closed Chain Function}

Since the foot is the foundational organ of the closed chain posture, it is of major importance that the foot be kept within its range of optimal functional positions as early as possible and for as long as possible. The primary thing we can provide a human foot with as far as its "optimal functional position" in closed chain is some qualitative position, $\mathrm{n}=1$, from which support and tasks can be performed most efficiently, injury and deformity free over its lifetime on a case to case basis as it morphs and changes its poses and axes $[16,17]$.

The current gold standard in biomechanics is a theoretical position known as subtalar joint neutral position and for me and others, it fails in two major areas. It fails to optimize the sagittal plane of the foot and it fails to represent the rearfoot segment of The Vault when seeking an optimal functional position. Forefoot examination, in the form of varus and valgus foot typing lacks independence and once again fails to optimize the front half of the three dimensional foot on the sagittal plane.

\section{Concession}

Because Biomechanics and Custom Foot Orthotics are lacking in consensus; language and terminology needed to be invented to begin to define the subject for discussion and further research and trial. I have conceived of and defined such language for consideration and review of the reader and welcome your comments and edits at this point.

\section{References}

1. McPoil TG, Cornwall MW (1994) Relationship between subtalar joint neutral position and rearfoot motion during walking. Foot Ankle Int 15(3): 141-145.

2. Payne CB (1998) The past, present, and future of podiatric biomechanics. JAPMA 88(2): 53-63. 
3. Lee WE (2001) Podiatric biomechanics. An historical appraisal and discussion of the Root model as a clinical system of approach in the present context of theoretical uncertainty. Clin Podiatr Med Surg 18(4): 555-684.

4. Nigg BM, Nurse MA, Stefanyshyn DJ (1999) Shoe inserts and orthotics for sport and physical activities. Med Sci Sports Exerc 31(Suppl 7): S421-S428.

5. Stacoff A, Reinschmidt C, Nigg BM, Bogert AJ, Lundberg A, et al. (2000) Effects of foot orthoses on skeletal motion during running. Clin Biomech (Bristol, Avon) 15(1): 54-64.

6. Heiderscheit B, Hamill J, Tiberio D (2001) A biomechanical perspective: do foot orthoses work? Br J Sports Med 35(1): 4-5.

7. Jarvis HL, Nestor CJ, Bowden PD, Jone RK (2017) Challenging the foundations of the clinical model of foot function: further evidence that the root model assessments fail to appropriately classify foot function. Jour of Foot \& Ankle Res 10: 7.

8. Bilanow T (2011) Do orthotics really help?

9. Pine J, Gilmore J (1998) Welcome to the experience economy. Harvard Business Review.
10. Foot Posture Theories. Orthopedia Wiki.

11. Kirby KA (2001) Subtalar joint axis location and rotational equilibrium theory of foot function. J Am Podiatr Med Assoc 91(9): 465-488.

12. Fuller EA, Kirby KA (2013) Subtalar joint equilibrium and tissue stress approach to biomechanical therapy of the foot and lower extremity. In: Albert SF, Curran SA (Eds.), Biomechanics of the Lower Extremity, Theory and Practice, Bipedmed, LLC, Denver, Colorado, USA, 1: 205-264.

13. Kirby K (2015) Prescribing orthoses: Has tissue stress theory supplanted root theory? Podiatry Today 28: 36-44.

14. Kirby KA, Spooner SK, Scherer PR, Schuberth JM (2012) Foot orthoses. Foot \& Ankle Specialist 5(5): 334-343.

15. Shavelson D (2007) A closer look at neoteric biomechanics. Podiatry Today 20(9): 60-66.

16. Root M, Orien W, Weed J (1977) Normal and abnormal function of the foot-Clinical biomechanics, volume 2. Physical Therapy 59(3): 352

17. Peacock D (2015) The peacock press test. Podiatry Management, pp. 138-144.
Creative Commons Attribution 4.0

International License

For possible submissions Click Here

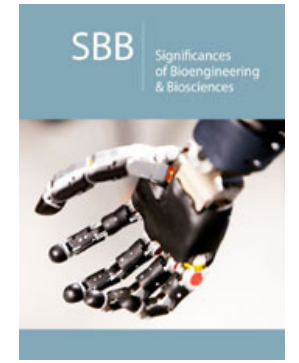

Significances of Bioengineering \& Biosciences

\section{Benefits of Publishing with us}

- High-level peer review and editorial services

- Freely accessible online immediately upon publication

- Authors retain the copyright to their work

- Licensing it under a Creative Commons license

- Visibility through different online platforms 